Suéllyn Mattos de Aragão ${ }^{1}$ André Luis Cândido da Silva ${ }^{1}$ Rafael Gomes Ditterich ${ }^{1}$ Solena Ziemer Kusma ${ }^{1}$

\title{
RECLAMATÓRIAS EM SAÚDE RECEBIDAS PELO MINISTÉRIO PÚBLICO DO ESTADO DO PARANÁ: UM ESTUDO DO CAOP DE PROTEÇÃo À SAÚdE PÚBLICA
}

Health-related claims made at the Public Prosecutor's Office of the State of Paraná: A study of the Operational Support Center of the Public Health Protection Prosecution Office

${ }^{1}$ Universidade Federal do Paraná. Curitiba/PR, Brasil.

Correspondência: Suéllyn Mattos de Aragão.E-mail: smdaragao@mppr.mp.br

Recebido: 31/05/2018. Revisão: 24/10/2018. Aprovado: 31/10/2018. 


\section{RESUMO}

O objetivo do estudo foi evidenciar os principais desafios sanitários no Estado do Paraná nos anos de 2016 e 2017, a partir da análise das denúncias realizadas junto ao Ministério Público Estadual. A proposta foi instrumentalizar gestores e usuários do sistema público para o reconhecimento $\mathrm{e}$ o enfrentamento das necessidades prementes em saúde. Realizou-se um estudo, documental e qualitativo, por meio da organização e análise de dados do sistema de informação do Centro de Apoio Operacional das Promotorias de Justiça de Proteção à Saúde Pública. Na categorização, adotou-se cinco classes iniciais, preconizadas pelo serviço estudado. Posteriormente, procedeu-se ao reconhecimento das categorias finais correspondentes e à formulação de conceitos norteadores com base nos fundamentos do Direito Sanitário. Os resultados demonstraram predomínio de demandas pertencentes à categoria relacionada a medicamentos, insumos e procedimentos. Destacou-se, também, a categoria referente às ocorrências de má prática profissional. A pesquisa concluiu que a principal fragilidade do Sistema Único de Saúde no Estado do Paraná, denunciada ao Ministério Público estadual, foi o provimento de medicamentos. Com a constatação de que a problemática dominante apontada pelo estudo relaciona-se ao fornecimento de fármacos e, por conseguinte, às limitações da universalidade e da integralidade do sistema público sanitário, foram debatidas as variantes imbricadas nesses preceitos: indefinição sobre os limites do dever do Estado, no que se refere ao oferecimento de serviços e produtos; extensão e pretensão da política de assistência farmacêutica do Sistema Único de Saúde; interesses conflitantes entre Estado e mercado; desafios regulatórios; entre outros.

\section{Palavras-Chave}

Assistência Farmacêutica; Integralidade em Saúde; Judicialização da Saúde; Ministério Público.

\section{ABSTRACT}

This study aims at highlighting the main challenges in public health in the state of Paraná during 2016 and 2017, through the analysis of claims made to the state Public Prosecutor' Office. The proposal is to provide instruments to public health care managers and users to recognize and confront pressing health needs. It was carried out a qualitative and documentary study through the organization and analysis of data from the information system of theOperational Support Center of the Public Health Protection Prosecution Office. In the categorization, five initial classes were adopted, indicated by the service studied. Subsequently, the corresponding final categories were recognized and guiding concepts were formulated, based on the foundations of the Health Law. The results showed a predominance of claims related to requests for medicines, supplies and procedures. The category referring to occurrences of bad professional practice was also remarkable. The study concluded that the main weakness in the state of Paraná, reported to the Public Prosecutor, was the supply of medicines. With the finding that the dominant problem pointed out by the study is related to the supply of drugs and, consequently, to the limitations of the universality and integrality of the public health system, the variants imbricated in these precepts were discussed: lack of definition regarding the boundaries of the State's duty of offering services and products; the extent and claim of pharmaceutical assistance policy of the Brazilian National Health System; conflicting interests between the State and the market; regulatory challenges, among others.

\section{Keywords}

Pharmaceutical Assistance; Integrality in Health; Judicialization of Health; Public Ministry. 


\section{Introdução}

A saúde é valor preponderante em sociedade. Consta da vigente ordem constitucional expressa previsão de que tal bem possui caráter de direito social, sendo atribuído ao Estado o dever de garanti-lo a todos os cidadãos mediante políticas públicas.

De acordo com Globekner ${ }^{1}$, inobstante tais disposições, o direito à saúde segue enfrentando desafios a sua concretização. Isso decorre dos fatores dinâmicos que o conformam, entre os quais estão a evolução das tecnologias em saúde e o progresso da organização da colaboração social, tanto no âmbito das relações privadas e do mutualismo que rege o sistema de saúde suplementar quanto no campo da organização do Estado como agente controlador, regulador e fomentador da atividade privada relacionada aos serviços sanitários.

Dessas dificuldades resultam, por exemplo, a consabida insatisfação quanto à morosidade no agendamento de consultas e exames, as limitações da assistência farmacêutica, a insuficiência de leitos hospitalares e a gestão deficitária do sistema. Tais elementos restringem os princípios da universalidade e da integralidade estabelecidos na Carta Magna e, frequentemente, alicerçam os pilares da judicialização sanitária.

A efetivação do Sistema Único de Saúde(SUS) como política de Estado e a concretização de uma saúde pública responsável e abrangente são objetos de um processo permanente que envolve diferentes atores e esforços conjuntos. A eficiência das soluções propostas pelas políticas sanitárias pressupõe, como decorrência direta dessa engrenagem, o envolvimento pleno desses agentes, conforme entendimento de Focault ${ }^{2}$, Noronha et al. ${ }^{3}$ e Paim ${ }^{4}$.

Por óbvio, a responsabilização pela coordenação do sistema público de saúde é do Estado, contudo não se pode olvidar que a lei também exige a participação, nesse processo de implementação, dos trabalhadores da saúde, dos movimentos sociais e sindicais, das equipes de auditoria, dos usuários, dos prestadores de serviço dos subsistemas complementar e suplementar, do Poder Legislativo e, no ponto que interessa a este trabalho, do Ministério Público (MP) ${ }^{5,6,7,8}$.

\footnotetext{
${ }^{1}$ GLOBEKNER, Osmir Antonio. A tutela individual e coletiva do direito à saúde pelo Ministério Público. Revista Jurídica da Escola Superior do Ministério Público de São Paulo, v. 8, p. 129-148, 2015. Disponível em: http://www.esmp.sp.gov.br/revista_esmp/index.php/RJESMPSP/article/download/209/120.

${ }^{2}$ FOCAULT, Michel. Microfísica do poder. 24. ed. São Paulo: Edição Graal, 2007.

${ }^{3}$ NORONHA, José Carvalho de; LIMA, Luciana Dias de; MACHADO, Cristiani Vieira. O Sistema Único de Saúde - SUS. In: GIOVANELLA, Ligia et al (Orgs.). Políticas e sistemas de saúde no Brasil. Rio de Janeiro: Ed. FIOCRUZ, 2012. p. 365-393.

${ }^{4}$ PAIM, Jairnilson Silva. Uma análise sobre o processo da Reforma Sanitária brasileira. Saúde em Debate, Rio de Janeiro, v. 33, n. 81, p. 27-37, jan./abr. 2009. Disponível em: https://repositorio.ufba.br/ri/bitstream/ ri/5978/1/Paim\%20JS\%202009.\%20Artigo2.pdf.

${ }^{5}$ REZENDE, Conceição Aparecida Pereira; PEIXOTO, Maria Passos Barcala. Metodologia para análises funcionais da gestão de sistemas e redes de serviços de saúde no Brasil. 1. ed. Brasília-DF: Organização Pan-Americana da Saúde (OPAS), 2003. v. 1.

${ }^{6}$ ALMEIDA, Gregório Assagra de; SOARES JÚNIOR, Jarbas; ASSIS, Gilmar de (Orgs.). Ministério Público e direitos fundamentais: saúde. Belo Horizonte: Del Rey, 2013.

${ }^{7}$ DALLARI, Sueli Gandolfi. Aspectos particulares da chamada judicialização da saúde. Revista de Direito Sanitário, São Paulo, v. 14, n. 1, p. 77-81, jun. 2013. ISSN 2316-9044. https://doi.org/10.11606/issn.23169044.v14i1p77-81. Disponivel em: http://www.revistas.usp.br/rdisan/article/view/56624.

${ }^{8}$ ASSIS, Gilmar de. SUS para todos: breves reflexões jurídico-sociais: avanços e desafios. ALMEIDA, Gregório Assagra de; SOARES JÚNIOR, Jarbas; ASSIS, Gilmar de (Orgs.). Ministério Público e direitos fundamentais: saúde. Belo Horizonte: Del Rey, 2013.
} 
Conforme previsão constitucional, o MP é uma instituição permanente, essencial à atuação jurisdicional do Estado, incumbido de defender a ordem jurídica, o regime democrático e os interesses sociais e individuais indisponíveis. Vale destacar que essa importante função de defesa dos direitos sociais lhe foi atribuída mais recentemente, pela Constituição Federal de $1988^{\circ}$ (CF/88). Além disso, uma de suas funções é zelar pelo efetivo respeito dos direitos assegurados na Constituição pelos poderes públicos e serviços de relevância pública, promovendo as medidas necessárias a sua garantia. Para tanto, o MP é dotado de especiais prerrogativas para a defesa de interesses individuais e da coletividade, principalmente os indisponíveis e os de longa abrangência social. Desse modo, tendo a Constituição definido a saúde como serviço de relevância pública e sendo o MP o legítimo defensor da sociedade, a instituição possui uma das atuações mais destacadas em salvaguardar o interesse maior das pessoas - a vida, direito difuso por excelência ${ }^{10}$.

Portanto, no entendimento de estudiosos como Chieffi ${ }^{11}$, Rotunno ${ }^{12}$, Maggio $^{13}$, Gomes ${ }^{14}$, Paula ${ }^{15}$ e Sarlet $^{16}$, o MP figura como importante agente no processo de implementação do SUS e como instrumento regulador das práticas estabelecidas, compelindo o Estado (gênero) a cumprir os postulados constitucionais e infraconstitucionais de proteção à saúde, sobretudo pelos instrumentos judiciais e extrajudiciais de que dispõe capazes de contribuir para o adequado funcionamento do sistema.

${ }^{9}$ BRASIL. Constituição da República Federativa do Brasil de 1988. Disponível em: http://www.planalto.gov. br/ccivil_03/constituicao/constituicaocompilado.htm. Acesso em: 25 set. 2019.

${ }^{10}$ RODRIGUES, João Gaspar. A nova dinâmica resolutiva do Ministério Público. Jus Navegandi, ago.2014. Disponível em: https://jus.com.br/artigos/30584/a-nova-dinamica-resolutiva-do-ministerio-publico. Acesso em: 08 mar. 2018.

${ }^{11} \mathrm{CHIEFFI,} \mathrm{Ana} \mathrm{Luiza;} \mathrm{BARATA,} \mathrm{Rita} \mathrm{Barradas.} \mathrm{Ações} \mathrm{judiciais:} \mathrm{estratégia} \mathrm{da} \mathrm{indústria} \mathrm{farmacêutica} \mathrm{para}$ introdução de novos medicamentos. Rev. Saúde Públ., São Paulo, v. 44, n. 3, p. 421-429, 2010. http://dx.doi. org/10.1590/S0034-89102010000300005. Disponível em: http://www.scielo.br/pdf/rsp/v44n3/05.pdf.

${ }^{12}$ ROTUNNO, Angela Salton. Judicialização e juridicização da saúde no Ministério Público. In: ASENSI, Felipe Dutra; PINHEIRO, Roseni (Orgs.). Direito sanitário. Rio de Janeiro: Elsevier, 2012, p. 517-531.

${ }^{13}$ MAGGIO, Marcelo P. A contribuição do Ministério Público à resolutiva implantação de políticas públicas de saúde: enfoque extrajudicial. Revista Jurídica do Ministério Público do Estado do Paraná, v. 1, p. 1-34, 2014. Disponível em: https://www.academia.edu/20947468/A_CONTRIBUI\%C3\%87\%C3\%830_ DO_MINIST\%C3\%89RIO_P\%C3\%9ABLICO_\%C3\%80_RESOLUTIVA_IMPLANTA\%C3\%87\%C3\%830_DE_ POL\%C3\%8DTICAS_P\%C3\%9ABLICAS_DE_SA\%C3\%9ADE_ENFOQUE_EXTRAJUDICIAL_1.

${ }^{14}$ GOMES, Valéria Bastos; PASSOS, Daniela Veloso Souza. A judicialização da saúde e as políticas públicas para fornecimento de medicamentos: uma análise a partir das decisões do TRF da 5 Região. Brazilian Journal of Empirical Legal Studies, Rio de Janeiro, v. 4, 2017. http://dx.doi.org/10.19092/reed.v4i3.168. Disponível em: https://www.researchgate.net/publication/321657139_A_JUDICIALIZACAO_DA_SAUDE_E_ AS_POLITICAS_PUBLICAS_PARA_FORNECIMENTO_DE_MEDICAMENTOS_UMA_ANALISE_A_PARTIR_DAS_ DECISOES_DO_TRF_DA_5_REGIAO.

${ }^{15}$ PAULA, Cléria Maria Lobo; BITTAR, Cléria Maria Lobo. Judicialização da saúde e seus reflexos na gestão do sistema único de saúde (SUS). Revista de Direitos Humanos e Efetividade, v. 3, p. 19-41, 2017. Disponível em: https://www.researchgate.net/publication/322100237_Judicializacao_da_saude_e_seus_reflexos_ na_gestao_do_Sistema_Unico_de_Saude_SUS.

${ }^{16}$ SARLET, Ingo Wolgang; SAAVEDRA, Giovani Agostini. Judicialização, reserva do possível e compliance na área da saúde. Revista de Direitos e Garantias Fundamentais, Vitória, v. 18, n. 1, p. 257-282, jan./abr. 2017. 
Por essa razão, faz-se importante o reconhecimento das reclamatórias sanitárias recebidas pelo ente, uma vez que elas constituem um indício inequívoco das fragilidades e deficiências do sistema público de saúde vigente. Tendo em vista as relações entre o órgão e o campo sanitário, e considerando, sobretudo, a existência de diversas modalidades e ramificações organizacionais, o presente estudo faz um recorte em tal estrutura tomando como seu objeto o Ministério Público do Estado do Paraná (MPPR), em especial seu Centro de Apoio Operacional das Promotorias de Justiça de Proteção à Saúde Pública (CAOP de Proteção à Saúde Pública) ${ }^{17}$, departamento de referência na instituição para assuntos relativos à matéria.

Com vistas a mapear os requerimentos, facilitar a consulta e divulgar o serviço prestado, o presente estudo identificou e analisou os principais desafios e limites do setor sanitário no Paraná, a partir da categorização das demandas relacionadas à saúde recebidas pelo Setor Médico do CAOP Saúde Pública, departamento do Ministério Público do Estado.

\section{Método}

Trata-se de um estudo documental, com abordagem qualitativa, realizado a partir da análise de conteúdo conforme preconizado pela metodologia de $\operatorname{Bardin}^{18}$, que, por meio da hermenêutica crítica, estrutura a elaboração e a análise de unidades de significado com o objetivo de sistematizar a captura da essência do fenômeno. A técnica consiste em examinar o material selecionado pelo pesquisador, buscando classificá-lo em temas ou categorias que auxiliam na compreensão da essência do objeto, para além do que está posto ${ }^{19}$. Dessa forma, um amplo e complexo conjunto de dados foi utilizado como base, com o intuito de gerar elementos manipuláveis, estabelecer relações e inferir conclusões ${ }^{20}$.

Bardin $^{21}$ assevera que a categorização é uma operação de classificação de elementos constitutivos de um conjunto, primeiro por diferenciação e, seguidamente, por reagrupamento por gênero (analogia, com os critérios previamente definidos). Segundo a autora, a análise faz-se principalmente por classificação-indexação e por intermédio de procedimentos de transformação, tendo como objetivo analisar e representar de forma condensada as informações provenientes dos elementos pesquisados, permitindo a elaboração de um documento secundário composto pelo máximo de informações sobre o objeto em questão. É precisamente esse procedimento que vem dotar a pesquisa de

\footnotetext{
${ }^{17}$ PARANÁ. Ministério Público do Estado. Resolução n 1.014, de 15 de agosto de 1997. Diário Oficial do Estado do Paraná, Curitiba, PR, 15 ago. 1997.

${ }^{18}$ BARDIN, Laurence. Análise de conteúdo. São Paulo: Edições 70, LDA, 2011.

${ }^{19} / d$. Ibid.

${ }^{20} / d$. Ibid.

${ }^{21}$ Id. Ibid.
} 
pertinência, viabilizando a compilação de denúncias recebidas pelo Ministério Público do Estado do Paraná em matéria de saúde de modo a torná-las acessíveis ao observador.

Inicialmente, foram tabuladas e analisadas duas planilhas do Microsoft Excel 2007 produzidas pelo setor médico do CAOP de Proteção à Saúde Pública, nominadas "Distribuição 2016" e "Distribuição 2017". Elas estão disponíveis na rede interna do setor e são utilizadas para controle e distribuição dos serviços. São emitidas anualmente e alimentadas diariamente, quando da entrada dos processos. Nelas, estão contidas as informações básicas dos procedimentos tramitados: código identificador, datas de entrada e saída no setor, categoria a que se refere a matéria e identificação do profissional responsável pela análise. Desse material foram extraídas as cinco categorias iniciais (macro). Tais variantes caracterizam os movimentos predominantes identificados na prática diária do serviço. Posteriormente, foram computadas as frequências absolutas e relativas respectivas dessas categorias. Os resultados foram individualizados para cada ano analisado.

Já de posse do código identificador (número do processo), foram buscados os pareceres técnicos referentes a cada um dos casos (total de 797). Esses documentos são emitidos por profissionais médicos, em média, de um a cinco dias após o estudo do processo, a depender da complexidade da análise. Estão registrados em arquivos do Microsoft Word 2007 e também se localizam na rede interna do serviço. Após a dupla leitura de cada relatório, foram identificadas as categorias finais (micro).

A partir disso, prosseguiu-se com a formulação de conceitos norteadores, à luz dos fundamentos teóricos do Direito Sanitário, para cada categoria final proposta. Finalmente, foram discutidos os aspectos técnico-jurídicos da categoria inicial de maior frequência, especialmente no que concerne aos preceitos da integralidade e da universalidade do SUS.

A população do estudo compõe-se de todas as demandas sanitárias recebidas pelo setor médico do CAOP de Proteção à Saúde Pública entre janeiro de 2016 de dezembro de 2017. Lançamentos incompletos (sem informação sobre o assunto reclamado) foram excluídos da pesquisa. O recorte temporal diz respeito ao marco dos 20 anos de fundação do Centro, referência na instituição em matéria sanitária, bem como ao período de agravamento da crise político-econômico-social do país. A coleta de dados ocorreu entre fevereiro e maio de 2018.

O estudo foi aprovado pelo Comitê de Ética em Pesquisa da Instituição de Ensino Superior participante, de acordo com a Resolução do Conselho Nacional de Saúde n. 466, de 12 de dezembro de 2012. 


\section{Resultados}

A pesquisa identificou e classificou 797 ocorrências recebidas entre 2016 e 2017 pelo Setor Médico do CAOP de Proteção à Saúde Pública. Apurou-se que as reclamatórias analisadas pertencem a cinco grandes grupos de categorias: (i) gestão do sistema público de saúde; (ii) leitos hospitalares; (iii) má prática profissional; (iv) medicamentos, insumos e procedimentos; e, finalmente, (v) saúde mental. A partir dos cinco eixos identificados, a dimensão analítica referente a medicamentos, insumos e procedimentos destacou-se entre as denúncias $(37,7 \%$ dos 797 casos, $\mathrm{n}=301$ ); a classe correspondente a má prática profissional também restou saliente ( $34 \%$ dos 797 casos, $n=271$ ), conforme disposto na Tabela 1 .

Tabela 1. Ocorrências recebidas pelo Setor Médico do Centro de Apoio Operacional das Promotorias de Proteção à Saúde Pública, 2016-2017

\begin{tabular}{lccccccc}
\hline Categorias iniciais & $\begin{array}{c}\text { Frequência } \\
\text { absoluta (n) }\end{array}$ & $\begin{array}{c}\text { Frequência } \\
\text { relativa (\%) }\end{array}$ & $\begin{array}{c}\text { Frequência } \\
\text { absoluta } \\
\text { biênio (n) }\end{array}$ & $\begin{array}{c}\text { Frequência } \\
\text { relativa } \\
\text { biênio (\%) }\end{array}$ \\
\hline & $\mathbf{2 0 1 6}$ & $\mathbf{2 0 1 7}$ & $\mathbf{2 0 1 6}$ & $\mathbf{2 0 1 7}$ & $\mathbf{2 0 1 6 / 2 0 1 7}$ & $\mathbf{2 0 1 6 / 2 0 1 7}$ \\
\hline I. Gestão do sistema público de saúde & 43 & 60 & 11,1 & 14,5 & 103 & 12,9 \\
II. Leitos hospitalares & 51 & 49 & 13,2 & 11,8 & 100 & 12,5 \\
III. Má prática profissional & 152 & 119 & 39,4 & 28,8 & 271 & 34,0 \\
IV. Medicamentos/ insumos/ procedimentos & 129 & 172 & 33,5 & 41,7 & 301 & 37,7 \\
V. Saúde mental & 10 & 12 & 2,5 & 2,9 & 22 & 2,7 \\
\hline Total & $\mathbf{3 8 5}$ & $\mathbf{4 1 2}$ & $\mathbf{4 8 , 3}$ & $\mathbf{5 1 , 6}$ & $\mathbf{7 9 7}$ & $\mathbf{1 0 0}$ \\
\hline & & & & & & Elaboração própria.
\end{tabular}

Os quadros apresentados a seguir ( 1 a 4 ) foram organizados de modo a enumerar as categorias finais em ordem decrescente de frequência.

Examinando o Quadro 1, relativo à categoria de gestão do sistema público de saúde, nota-se que as questões associadas às limitações no acesso e na cobertura, à baixa resolubilidade, à escassez de recursos humanos, às falhas nos serviços de urgência e emergência, às debilidades em linhas de cuidado específicas e aos crimes sanitários são as principais impropriedades noticiadas. Nesse grupo, as maiores reclamatórias dizem respeito às infindáveis filas de espera por atendimento. Outras temáticas também identificadas tratam de denúncias de irregularidades nas relações entre a esfera pública e a privada na engrenagem do SUS e de conflitos relacionados aos limites de competência e de responsabilidade dos entes na execução em saúde (municipal, estadual e federal). 
Quadro 1. Descrição da Categoria I - Gestão do sistema público de saúde

\begin{tabular}{|c|c|}
\hline Conceito norteador & Categoria final \\
\hline $\begin{array}{l}\text { - Persistência de desigualdades no acesso } \\
\text { ao sistema público de saúde } \\
\text { - Baixa resolubilidade }\end{array}$ & $\begin{array}{l}\text { 1. Morosidade no andamento das filas de espera } \\
\text { para atendimento nas especialidades médicas } \\
\text { (ortopedia, cirurgia geral, cirurgia plástica, cirurgia } \\
\text { bariátrica, pneumologia, oftalmologia, reprodução } \\
\text { assistida, cardiologia) [n=23] }\end{array}$ \\
\hline $\begin{array}{l}\text { - As limitações de cobertura de um sistema } \\
\text { de saúde dito universal }\end{array}$ & $\begin{array}{l}\text { 2. Restrições na cesta de exames ofertados por } \\
\text { Municípios ou Estado [ } n=19]\end{array}$ \\
\hline $\begin{array}{l}\text { - Dificuldades de Estado e Municípios na } \\
\text { contratação de profissionais de saúde, } \\
\text { agravadas pela conjuntura de Reforma do } \\
\text { Estado, com pressões para redução de } \\
\text { gastos com pessoal } \\
\text { - Aumento da precarização das relações de } \\
\text { trabalho na saúde }\end{array}$ & $\begin{array}{l}\text { 3. Escassez de recursos humanos em } \\
\text { estabelecimentos de saúde [n=11] }\end{array}$ \\
\hline $\begin{array}{l}\text { - A busca pelo rigor científico em áreas } \\
\text { correlatas à saúde }\end{array}$ & $\begin{array}{l}\text { 4. Pronunciamentos técnicos para instrução de } \\
\text { procedimentos administrativos [ } n=09]\end{array}$ \\
\hline $\begin{array}{l}\text { - Deficiências na qualidade e resolubilidade } \\
\text { da atenção em diferentes pontos do } \\
\text { sistema de saúde }\end{array}$ & $\begin{array}{l}\text { 5. Impropriedades no atendimento dos serviços } \\
\text { móveis de urgência }[n=07]\end{array}$ \\
\hline $\begin{array}{l}\text { - Inadequações nas condições sanitárias de } \\
\text { estabelecimentos de saúde }\end{array}$ & 6. Vistorias/inspeções em clínicas, hospitais $[n=07]$ \\
\hline $\begin{array}{l}\text { - Persistência da agenda das patologias } \\
\text { infectocontagiosas }\end{array}$ & $\begin{array}{l}\text { 7. Acompanhamento das medidas adotadas } \\
\text { por gestores frente a enfermidades específicas } \\
\text { (H1N1, hanseníase, tuberculose, infecção } \\
\text { hospitalar, dengue, febre amarela etc.) }[\mathrm{n}=06]\end{array}$ \\
\hline $\begin{array}{l}\text { - Deficiências nos sistemas e estações de } \\
\text { tratamento de água }\end{array}$ & $\begin{array}{l}\text { 8. Avaliações concernentes à qualidade da água } \\
\text { para consumo humano }[n=05]\end{array}$ \\
\hline - O crime no sistema público de saúde & 9. Cobranças indevidas no SUS [n=04] \\
\hline - O crime no sistema público de saúde & $\begin{array}{l}\text { 10. Ilicitudes entre a coisa pública e a privada em } \\
\text { saúde }[n=04]\end{array}$ \\
\hline $\begin{array}{l}\text { - A gestão deficitária, sob a ótica técnica } \\
\text { e política } \\
\text { - Controle social no SUS }\end{array}$ & $\begin{array}{l}\text { 11. Descumprimento de normativas do Ministério } \\
\text { da Saúde }[n=03]\end{array}$ \\
\hline $\begin{array}{l}\text { - A iniquidade e as políticas voltadas aos } \\
\text { vulneráveis na esfera do sistema público } \\
\text { de saúde }\end{array}$ & $\begin{array}{l}\text { 12. Apreciação de enquadramento para fins de } \\
\text { concessão de benefícios sociais (ex.: isenção } \\
\text { tarifária) }[n=02]\end{array}$ \\
\hline $\begin{array}{l}\text { - A complexidade dos fluxos de referência e } \\
\text { contrarreferência }\end{array}$ & $\begin{array}{l}\text { 13. Lógica organizativa deficitária dos serviços de } \\
\text { saúde }[n=02]\end{array}$ \\
\hline $\begin{array}{l}\text { - A responsabilidade compartilhada entre } \\
\text { entes federativos }\end{array}$ & $\begin{array}{l}\text { 14. Identificação da competência e } \\
\text { responsabilidade de cada ente federativo na } \\
\text { execução em saúde (municipal, estadual e } \\
\text { federal) [n=01] }\end{array}$ \\
\hline
\end{tabular}


Conforme disposto no Quadro 2, observa-se que, ante o esgotamento do fluxo ordinário de transferência de enfermos entre unidades de urgência e emergência e estabelecimentos possuidores de recursos de maior complexidade (nosocômios), usuários ou seus representantes têm buscado amparo no órgão ministerial a fim de assegurar a celeridade e a eficiência nos encaminhamentos.

Quadro 02. Descrição da Categoria II - Leitos hospitalares

\begin{tabular}{|c|c|}
\hline Conceitos norteadores & Categoria final \\
\hline $\begin{array}{l}\text { - Os óbices no acesso ao sistema público de saúde } \\
\text { - Conflitos na regulação }\end{array}$ & $\begin{array}{l}\text { 1. Morosidade na transferência de } \\
\text { pacientes que se encontram em Unidades } \\
\text { de Pronto Atendimento e necessitam de } \\
\text { transferência para leito hospitalar (clínico, } \\
\text { cirúrgico, psiquiátrico ou de UTI) [n=83] }\end{array}$ \\
\hline $\begin{array}{l}\text { - Os entraves nos fluxos de referenciamento de pacientes } \\
\text { e na lógica organizativa no sistema público de saúde } \\
\text { - As dissidências entre os gestores nas esferas } \\
\text { municipais, estaduais e federais } \\
\text { - Conflitos na regulação }\end{array}$ & $\begin{array}{l}\text { 2. Dificuldades no encaminhamento de } \\
\text { pacientes internados em instituições } \\
\text { desprovidas da capacidade técnica } \\
\text { necessária à resolução do caso [n=12] }\end{array}$ \\
\hline $\begin{array}{l}\text { - As prerrogativas de um sistema universal de saúde } \\
\text { - As dinâmicas territoriais em saúde } \\
\text { - Conflitos na regulação }\end{array}$ & $\begin{array}{l}\text { 3. Impasses nas transferências } \\
\text { intermunicipais de pacientes }[n=05]\end{array}$ \\
\hline
\end{tabular}

Fonte: Elaborado pelos autores

Destacam-se, no Quadro 3, as queixas de má prática técnica detectadas no universo de dados. As acusações estão relacionadas à suposta deficiência na qualidade da atenção ofertada e englobam, particularmente, contestações de diagnóstico (inexato ou tardio), má interpretação ou não solicitação de exames, dosagens medicamentosas e combinações farmacológicas impróprias, má técnica cirúrgica, não conferência de informações básicas, como o histórico de alergias, entre outros.

Os documentos analisados apontam 271 denúncias, envolvendo diferentes categorias profissionais (médicos, enfermeiros, auxiliares de enfermagem, fisioterapeutas, cirurgiões dentistas, entre outros).

Quadro 3. Descrição da Categoria III - Má prática profissional

\begin{tabular}{|c|c|}
\hline Conceitos norteadores & Categoria final \\
\hline $\begin{array}{l}\text { - A correta identificação da competência } \\
\text { legal de cada classe profissional }\end{array}$ & $\begin{array}{l}\text { 1. Verificações quanto à legalidade da prática profissional } \\
\text { (médicos, enfermeiros, fisioterapeutas etc.) }[n=126]\end{array}$ \\
\hline $\begin{array}{l}\text { - Os problemas referentes à qualidade } \\
\text { e resolubilidade da atenção ofertada }\end{array}$ & $\begin{array}{l}\text { 2. Apurações acerca da qualidade técnica do } \\
\text { atendimento prestado em casos envolvendo sequelas } \\
\text { ou óbito de pacientes [n=113] }\end{array}$ \\
\hline $\begin{array}{l}\text { - A correta identificação da competência } \\
\text { legal de cada classe profissional }\end{array}$ & $\begin{array}{l}\text { 3. Avaliações de competência técnica e legal para } \\
\text { realização de procedimentos e cirurgias [n=32] }\end{array}$ \\
\hline
\end{tabular}


O Quadro 4 apresenta informações acerca da categoria equivalente aos medicamentos, insumos e procedimentos disponibilizados pelo SUS, a principal categoria reclamada no universo estudado. Ante todos os fármacos e insumos solicitados $(\mathrm{n}=301)$, os mais frequentes, em ordem decrescente, foram: rivaroxabana $(7,6 \%$, $\mathrm{n}=23)$, brometo de tiotrópio $(6,3 \%, \mathrm{n}=19)$, denosumabe $(4,6 \%, \mathrm{n}=14)$ e teriparatida $(4,3 \%, n=13)$. É importante notar que, dentre os medicamentos pleiteados, muitos foram apreciados e receberam negativa da Comissão Nacional de Incorporação de Tecnologias no SUS (Conitec), instância criada com a Lei n. 12.401/201122 responsável por assessorar o Ministério da Saúde nas decisões de inclusão dos fármacos à Relação Nacional de Medicamentos Essenciais (Rename). As principais motivações alegadas para as recomendações de não incorporação foram fatores pertinentes à eficácia e à segurança, ao custo-efetividade e à ausência de comprovação de benefícios em relação às drogas já disponibilizadas pelo SUS ${ }^{23}$.

Quadro 4. Descrição da Categoria IV - Medicamentos/insumos/procedimentos

\begin{tabular}{|c|c|}
\hline Conceitos norteadores & Categoria final \\
\hline $\begin{array}{l}\text { - As limitações na assistência farmacêutica } \\
\text { do SUS } \\
\text { - Vazios assistenciais }\end{array}$ & $\begin{array}{l}\text { 1. Solicitações de medicamentos não } \\
\text { incorporados à Relação Nacional de } \\
\text { Medicamentos Essenciais (Rename) [n=143] }\end{array}$ \\
\hline $\begin{array}{l}\text { - As limitações na assistência farmacêutica } \\
\text { do SUS } \\
\text { - O avanço científico e o subfinanciamento } \\
\text { do sistema público de saúde }\end{array}$ & $\begin{array}{l}\text { 2. Requerimentos de medicamentos relacionados } \\
\text { à incorporação tecnológica (ainda em fase de } \\
\text { experimento ou já comercializados), não registrados } \\
\text { na Anvisa, manipulados, com necessidade de } \\
\text { importação, de alto custo etc. [ } n=51 \text { ] }\end{array}$ \\
\hline $\begin{array}{l}\text { - As limitações na cobertura do SUS } \\
\text { - O avanço científico e o subfinanciamento } \\
\text { do sistema público de saúde } \\
\text { - Dependência de importações em } \\
\text { diferentes setores do sistema }\end{array}$ & $\begin{array}{l}\text { 3. Pedidos de órteses, próteses, insumos (ex.: } \\
\text { bombas de insulina, tiras reagentes, stents, elevador } \\
\text { para paciente, oxímetros, respiradores, cadeiras de } \\
\text { rodas, frascos de dietas industrializadas, cânulas, } \\
\text { sondas, materiais para curativo, fraldas etc.) [n=43] }\end{array}$ \\
\hline $\begin{array}{l}\text { - O avanço científico e o subfinanciamento } \\
\text { do sistema público de saúde }\end{array}$ & 4. Petições de dietas industrializadas $[n=28]$ \\
\hline $\begin{array}{l}\text { - Defasagem tecnológica e dependência de } \\
\text { importações em vários segmentos do sistema }\end{array}$ & 5. Requerimentos de testes genéticos, exames $[n=19]$ \\
\hline A morosidade no sistema público de saúde & $\begin{array}{l}\text { 6. Solicitações de execução de procedimentos e } \\
\text { cirurgias }[n=12]\end{array}$ \\
\hline $\begin{array}{l}\text { O descompasso entre o incremento } \\
\text { tecnológico e o financiamento do sistema } \\
\text { público de saúde }\end{array}$ & $\begin{array}{l}\text { 7. Pleito por serviços especializados (home care, } \\
\text { enfermagem contínua) [n=05] }\end{array}$ \\
\hline
\end{tabular}

Fonte: Elaborado pelos autores

\footnotetext{
${ }^{22}$ BRASIL. Lei n. 12.401, de 28 de abril de 2011. Altera a Lei n. 8.080, de 19 de setembro de 1990, para dispor sobre a assistência terapêutica e a incorporação de tecnologia em saúde no âmbito do Sistema Único de Saúde - SUS. Disponível em: http://www.planalto.gov.br/ccivil_03/_Ato2011-2014/2011/Lei/ L12401.htm. Acesso em: 25 set. 2019.

${ }^{23}$ RELATÓRIOS de recomendação da Conitec. CONITEC. Comissão Nacional de Incorporação de Tecnologias no Sistema Único de Saúde, 29 jul. 2014. Disponível em: http://conitec.gov.br/relatorios-de-recomendacao-da-conitec.
} 
Para além dos requerimentos atinentes à assistência farmacêutica, situações alusivas à dificuldade de acesso ou à indisponibilidade de serviços e insumos foram identificadas, tais como: delongas para realização de procedimentos e cirurgias, petições de bomba de insulina, tiras reagentes, stents, elevador para paciente, oxímetros, respiradores, cadeiras de rodas, frascos de dietas industrializadas, cânulas, sondas, materiais para curativos, fraldas, câmara hiperbárica, CPAP (Continuous Positive Airway Pressure), dietas industrializadas, fertilização in vitro, imunoterapia, litotripsia, neuromodulação, implantes, próteses e órteses, entre outros.

No que concerne à classe de saúde mental, os assuntos mais frequentemente comunicados dizem respeito à política de anti-institucionalização, à nova rede de atenção (Centros de Atenção Psicossocial) e à Reforma Psiquiátrica. Nota-se que a saúde mental, embora tão preciosa à população e historicamente negligenciada pelo poder público, apresentou uma baixa frequência absoluta de denúncias $(2,7 \%$ dos 797 casos, $n=22$ ) comparativamente às demais categorias. Por hipótese, pode-se dizer que, no estado do Paraná, um campo da saúde que já foi muito preterido parece hoje atender mais satisfatoriamente à população do que em outros tempos. Todavia, para a confirmação desta suposição, uma pesquisa mais extensa seria necessária.

\section{Discussão}

Em razão dos limites do presente escrito e em virtude de as matérias ligadas ao fornecimento público de medicamentos, insumos e procedimentos terem se destacado como um dos principais achados da pesquisa, as discussões aqui apresentadas voltam-se a essa temática, constituindo-a no foco da análise.

Segundo Nakamura ${ }^{24}$, a judicialização da saúde brasileira decorre, em grande medida, da dificuldade que o Legislativo e o Executivo têm para se reorganizar permanentemente a fim de atender a multiplicidade de casos que se apresentam cotidianamente no âmbito do SUS, particularmente no que se refere à execução da política de medicamentos em cada esfera federativa.

Magalhães ${ }^{25}$ esclarece que o tema é multifacetado e envolve análises complexas, como: (i) a separação de poderes ou a legitimidade da ingerência do Poder Judiciário na definição e/ou execução de políticas públicas construídas politicamente; (ii) o princípio da isonomia, ou seja, o favorecimento dos cidadãos que demandam

\footnotetext{
${ }^{24}$ NAKAMURA, Fernanda de Castro. A judicialização da saúde e a atuação do Ministério Público no sistema de fornecimento gratuito de medicamentos na região de Ribeirão Preto. 2017. Dissertação (Mestrado em Planejamento a Análises de Políticas Públicas) - Universidade Estadual Paulista Júlio de Mesquita Filho, Franca, 2017.

${ }^{25}$ MAGALHÃES, lasmim Pacheco. Judicialização da saúde: critérios e limites para a atuação de magistrados em ações judiciais para aquisição de medicamentos pelo Estado. 2014. 65 f. Monografia (Bacharelado em Direito) - Universidade de Brasília, Brasília-DF, 2014. Disponível em: http://bdm.unb.br/ bitstream/10483/10100/1/2014_lasmimPachecoMagalhaes.pdf.
} 
prestações de saúde judicialmente em relação aos que apenas aguardam atendimento pelo SUS; e (iii) a possibilidade de limitações no exercício do direito à saúde, enlaçada ao princípio da reserva do financeiramente possível, que culmina na embaraçosa ponderação de bens ou valores constitucionais.

Nessa sistemática, a ordem judicial e seus prepostos podem ser considerados tanto um sistema legal dentro do qual os minoritários exercem seus direitos, como um espaço com potencial de desservir a saúde como um bem coletivo. No entanto, ao endossar reivindicações incalculáveis, dentro de um cenário de recursos finitos, há risco de se comprometer, de maneira notável, o funcionamento global do sistema ${ }^{26}$. Essa tem sido uma das principais críticas à interferência do Judiciário nas decisões em saúde: o desregramento dos gastos e o eventual descontrole do equilíbrio financeiro, imediato e tardio, do sistema público de saúde.

Sobre a matéria, Souza ${ }^{27}$ constata que a insistência do Judiciário brasileiro em adjudicar bens públicos individualizados, ao invés de determinar a implantação das políticas adequadas, tem levado à predação da renda pública pelas elites, a exemplo do que acontece em outros países. Nesse contexto, há que se ressaltar que a desigualdade de oportunidade de acesso à justiça é fator incontroverso. Muito embora existam defensores públicos, organizações de assistência judiciária sem fins lucrativos e convênios entre a Ordem dos Advogados do Brasil (OAB) e os estados, as populações pertencentes às classes mais baixas de renda e as minorias (mulheres, negros, população LGBT, indígenas, deficientes) encontram, do ponto de vista fático, maiores obstáculos quando da necessidade de acionamento do Poder Judiciário.

No que declaram Oliveira ${ }^{28}$ e Costa e Borges ${ }^{29}$, a conquista do direito à saúde não terminou com sua inscrição na Carta Magna. Os autores argumentam que os "tijolos assentados" até agora para sua implementação, embora tenham representado um enorme avanço, não foram suficientes para levantar a "morada" desse direito para todos. Sustentam, sobretudo, que, enquanto houver indicadores sociais a demonstrar iniquidades, injustiça social e quadros epidemiológicos não favoráveis, o direito à saúde permanece em construção.

De fato, a retórica da propositura de ações judiciais relacionadas ao direito à saúde em face do poder público permite diferentes perspectivas de observação.

\footnotetext{
${ }^{26}$ COSTA, A.C.; BORGES, M.S. A judicialização como acesso ao direito à saúde: considerações ao debate brasileiro. Sociedade em Debate, Pelotas, v. 16, n. 1, p. 77-89, 2012.

${ }^{27}$ SOUZA NETO, Claudio Pereira de; SARMENTO, Daniel (Coords.). Direitos sociais: fundamentos, judicialização e direitos sociais em espécie. Rio de Janeiro: Lumen Juris, 2008. p. 313-339.

${ }^{28}$ OLIVEIRA, Maria dos Remédios Mendes et al. Judicialização da saúde: para onde caminham as produções científicas? Saúde debate [online], Rio de Janeiro, v. 39, n. 105, p. 525-535, 2015. http:// dx.doi.org/10.1590/0103-110420151050002019. Disponível em: http://www.scielo.br/pdf/sdeb/ v39n105/0103-1104-sdeb-39-105-00525.pdf.

${ }^{29} \mathrm{COSTA}$, Alexandre Bernardino et al. O Direito achado na rua: introdução crítica ao direito à saúde. BrasíliaDF: CEAD/ UnB, Brasília, 2009. p. 103-111.
} 
No entanto, levantamentos ${ }^{30}$ indicam que grande parte do passivo judicializado concentra-se no campo do fornecimento de medicamentos e insumos. Essas ações representaram um boom na litigação dos direitos fundamentais e trouxeram, para o âmbito judicial, a discussão sobre a incapacidade de o Poder Público implementar políticas que realmente atendam a todos, indiscriminadamente.

Machado $^{31}$ e Figueiredo $^{32}$ denotam que tal modalidade de demanda, iniciada na década de 1990 a partir de solicitações de drogas antirretrovirais para o combate do HIV/AIDS, tem apresentado um papel importante como via alternativa ao acesso a fármacos, insumos e procedimentos no âmbito do sistema estatal. Algumas características comuns a esses requerimentos são identificadas em diferentes estudos - por exemplo, a maioria dos pedidos é individual, e as prescrições são tanto de medicamentos incorporados como de não incorporados pelo SUS, alguns sem registro no país ou em indicação terapêutica não constante do registro sanitário.

Há um incremento exponencial nos valores despendidos com essas solicitações. Machado et al. ${ }^{33}$ demonstram que, em 2005, o governo federal gastou diretamente R \$2,5 milhões com aquisição de medicamentos solicitados pela via judicial e que o ente foi citado como réu em 387 processos. Em 2007, o gasto passou para $\mathrm{R} \$ 15$ milhões destinados ao atendimento de aproximadamente três mil ações. Em 2008, as despesas alcançaram R 52 milhões. Outros estudos demonstram gastos crescentes também por parte dos estados e dos municípios, gerando preocupação entre os gestores ${ }^{34}$.

Ao discutir a questão, $\mathrm{Smith}^{35}$ sugere que também deve ser considerada a questão do conflito de interesses incorporado ao trinômio judicialização, profissionais de saúde e indústria. Ao demais, há uma fragilidade incontestável nas demandas jurídicas em saúde: o discurso médico e sua incontrastabilidade. Isso porque o

${ }^{30}$ PEPE, V. L. E. et al. A judicialização da saúde e os novos desafios da gestão da assistência farmacêutica. Ciência e Saúde Coletiva, (Impresso), v. 15, n. 5, p. 2405-2414, 2010.

${ }^{31}$ MACHADO, Marina Amaral de Ávila. Acesso a medicamentos via poder judiciário no Estado de Minas Gerais. 2010. Dissertação (Mestrado) - Faculdade de Farmácia, Universidade Federal de Minas Gerais, Belo Horizonte-MG, 2010.

${ }^{32}$ FIGUEIREDO, Tatiana Aragão. Análise dos medicamentos fornecidos por mandado judicial na Comarca do Rio de Janeiro: a aplicação de evidências científicas no processo de tomada de decisão. 2010. Dissertação (Mestrado em Saúde Pública). Escola Nacional de Saúde Pública Sérgio Arouca. Fundação Oswaldo Cruz, Rio de Janeiro, 2010.

${ }^{33}$ MACHADO Marina Amaral de Ávila et al. Judicialização do acesso a medicamentos no Estado de Minas Gerais, Brasil. Rev. saúde pública, v. 45, n. 3, p. 590-598, 2011. http://dx.doi.org/10.1590/S003489102011005000015. Disponivel em: http://www.scielo.br/pdf/rsp/v45n3/2403.pdf.

${ }^{34}$ LEITE, Silvana Nair; MAFRA, Ana Cristina Que direito? Trajetórias e percepções dos usuários no processo de acesso a medicamentos por mandados judiciais em Santa Catarina. Ciênc. saúde coletiva [online], v. 15, n. 1, p. 1665-1672, 2010. http://dx.doi.org/10.1590/S1413-81232010000700078. Disponível em: http://www.scielo.br/pdf/csc/v15s1/078.pdf.

${ }^{35} \mathrm{SMITH}$ Richard. Medical journals are an extension of the marketing arm of pharmaceutical companies. PLoSMed, v. 2, n. 5, p. 138, 2015. https://doi.org/10.1371/journal.pmed.0020138. Disponível em: https://journals.plos.org/plosmedicine/article/file?id=10.1371/journal.pmed.0020138\&type=printable. 
Judiciário não possui condições de contrastar plenamente o que se pede, tendo em vista sua impossibilidade de se apropriar integralmente do caso em razão da insuficiência de conhecimento técnico. Entretanto, a criação de grupos técnicos de suporte com a finalidade de subsidiar decisões da magistratura, como os Núcleos de Apoio Técnico (NAT), adstritos a diferentes instâncias forenses, tem sido um contraponto.

É importante ponderar que, de acordo com o contido no artigo 194, inciso I, da $\mathrm{CF} / 88$, um dos pilares a seguridade social é a universalidade da cobertura e do atendimento. A integralidade, muito embora apareça categoricamente no artigo 198 da CF/88, inciso II, como uma diretriz do SUS, pode ser interpretada simultaneamente como um princípio, tendo em vista o artigo $7^{\circ}$ da Lei n. 8.080/1990 ${ }^{36}$. Desse modo, sua natureza jurídica é tida como híbrida, sendo sincronicamente um princípio e uma diretriz do SUS. Seja como princípio, seja como diretriz, é notório que seus contornos jurídicos são, ainda, bastante imprecisos, cercados de controvérsias hermenêuticas que afetam o sistema jurídico-sanitário e que carecem de melhor elaboração $0^{37,38}$.

Segundo Gomes ${ }^{39}$ e Mello ${ }^{40}$, a indefinição sobre os horizontes do dever do Estado, no que se refere ao oferecimento integral de serviços e produtos, contribui para o incremento do litígio da saúde no Brasil. Asensi ${ }^{41}$, Cardoso ${ }^{42}$ e Loureiro ${ }^{43}$ citam o provisionamento insuficiente de produtos, a solicitação de medicamentos não padronizados e/ou a demora no processo de inclusão de fármacos e insumos nas listas públicas como outros fatores significativos.

${ }^{36}$ BRASIL. Lei n. 8.080, de 19 de setembro de 1990. Dispõe sobre as condições para a promoção, proteção e recuperação da saúde, a organização e o funcionamento dos serviços correspondentes e dá outras providências. Disponível em: http://www.planalto.gov.br/ccivil_03/leis/L8080.htm. Acesso em: 25 set. 2019.

${ }^{37}$ AITH, Fernando; BUJDOSO, Yasmin; NASCIMENTO, Paulo Roberto do; DALLARI, Sueli Gandolfi. Os princípios da universalidade e integralidade do SUS sob a perspectiva da política de doenças raras e da incorporação tecnológica. Revista de Direito Sanitário, v. 15, p. 10, jul. 2014. https://doi.org/10.11606/issn.23169044.v15i1p10-39. Disponível em: http://www.revistas.usp.br/rdisan/article/view/82804/85759.

${ }^{38}$ BRITO-SILVA, Keila; BEZERRA, Adriana Falangola Benjamin; TANAKA, Oswaldo Yoshimi. Direito à saúde e integralidade: uma discussão sobre os desafios e caminhos para sua efetivação. Interface (UNI/UNESP. Online), v. 16, n. 40, p. 249-260, 2012. http://dx.doi.org/10.1590/S1414-32832012005000014. Disponível em: http://www.scielo.br/pdf/icse/v16n40/aop1812.pdf.

${ }^{39}$ GOMES, Valéria Bastos; PASSOS, Daniela Veloso Souza. op. cit.

${ }^{40}$ MELLO, Alexandra Ferro de et al. Uma abordagem econômica de processos judiciais de medicamentos impetrados contra um município do sul do Brasil. Jornal Brasileiro de Economia da Saúde, v. 8, n. 1, p. 39-46, 2016. http://dx.doi.org/ 10.21115/JBES.v8.n1.p39-46. Disponível em: http://files.bvs.br/ upload/S/2175-2095/2016/v8n1/a5479.pdf.

${ }^{41}$ ASENSI, Felipe Dutra. Indo além da judicialização: o ministério público e a saúde no Brasil. Rio de Janeiro, RJ: Escola de Direito do Rio de Janeiro da Fundação Getúlio Vargas, Centro de Justiça e Sociedade, 2010. Disponível em: http://bibliotecadigital.fgv.br/dspace/handle/10438/10313.

${ }^{42}$ CARDOSO, Cosme Santana. Análise das demandas judiciais de medicamentos: uma abordagem da realidade atual do Distrito Federal. Revista Jurídica da Procuradoria-Geral do Distrito Federal, Distrito Federal, Brasília-DF, v. 39, n. 1, p. 11-30, 2014. Disponível em: http://revista.pg.df.gov.br/index.php/ RJPGDF/article/download/210/133.

${ }^{43}$ LOUREIRO, Maria Rita. As origens e consequências da judicialização da política. Resenha. Revista Brasileira Ciência Sociais, São Paulo, v. 29, n. 84, p. 189-199, fev. 2014. http://dx.doi.org/10.1590/ S0102-69092014000100012. Disponível em: http://www.scielo.br/pdf/rbcsoc/v29n84/11.pdf. 
A priori, parte-se da platitude de que os princípios constitucionais e regimentais garantiriam aos pacientes, ao menos em tese, o direito a todos os serviços médicos no país, inexistindo uma definição explícita da inclusão e/ou da exclusão de produtos, procedimentos e cuidados que são de responsabilidade da oferta pública ${ }^{44,45}$.

$\mathrm{Na}$ contracorrente, alguns autores ${ }^{46}$ defendem a criação de uma cesta de serviços a ser ofertada pelo sistema público a todos os cidadãos como forma de alcançar os princípios da universalidade e, ao mesmo tempo, equidade. A definição do pacote se basearia em três critérios básicos: análises de custo-efetividade, estudos de evidência clínica e análises de impacto orçamentário.

Nessa toada, a Primeira Seção do Superior Tribunal de Justiça (STJ) concluiu, em decisão inédita no julgamento do Recurso Repetitivo n. 1.657.156, publicado em 05 de maio de $2018^{47}$, pela fixação de requisitos para que o Poder Judiciário determine o fornecimento de fármacos não constantes na lista do SUS. A deliberação incide em processos distribuídos após a publicação do escrito. Os critérios definidos incluem a incapacidade financeira do paciente de arcar com o custo do medicamento prescrito e a existência de registro do medicamento na Agência Nacional de Vigilância Sanitária (Anvisa). Ainda não é possível antever quais serão os efeitos da decisão no contexto da judicialização farmacêutica, mas o cenário aponta para restrições de direitos. A comunidade jurídica poderá questionar, inclusive, o contraste da decisão com os preceitos de universalidade, integralidade e equidade do sistema público de saúde.

Inobstante tal cenário, corrente majoritária de pensadores do Direito Sanitário ampara o conquistado até aqui: uma saúde gratuita, universal e integral para todos. Conquanto, a trajetória e o tempo vivido são permeados de desafios que se contrapõem ao estatuto constitucional ${ }^{48}$.

Nas palavras de Tanaka ${ }^{49}$, o direito à saúde universal, integral e equitativa é, sem dúvida, um ganho em nosso processo democrático. A lógica contributiva

\footnotetext{
${ }^{44}$ CANUT, L. Operacionalização do Sistema Único de Saúde e de sua assistência farmacêutica diante da judicialização: um estudo de caso no Município de São José/SC. Revista de Direito Sanitário, v. 18, n. 2, p. 62-91, 26 dez. 2017. https://doi.org/10.11606/issn.2316-9044.v18i2p62-91. Disponível em: https:// www.revistas.usp.br/rdisan/article/view/142012.

${ }^{45}$ MAGGIO, Marcelo P. Tutela da saúde pública: novas perspectivas e a construtiva atuação do Ministério Público. 2018. Tese (Doutorado em Serviços de Saúde Pública) - Faculdade de Saúde Pública, Universidade de São Paulo, São Paulo, 2018. Disponível em: http://www.teses.usp.br/teses/disponiveis/6/6135/tde-12042018-123251/.

${ }^{46}$ ANDRADE, Mônica Viegas; NORONHA, K. V. M. S. Uma nota sobre o princípio da integralidade do SUS. In: BACHA, Edmar; SCHWARTZMAN, Simon (Orgs.). Brasil: a nova agenda social. Rio de Janeiro: LTC, 2010. v. 1, p. 94-103.

${ }^{47}$ SUPERIOR TRIBUNAL DE JUSTIÇA - STJ. Recurso Especial: REsp n. 1.657.156 - RJ 2017/0025629-7. Primeira Seção. Relator: Ministro Benedito Gonçalves, julgado em 25 de abril de 2018, publicado em 04 de maio de 2018. Disponível em: https://ww2.stj.jus.br/processo/pesquisa/?aplicacao=processos.ea\&tip oPesquisa $=$ tipoPesquisaGenerica\&termo=REsp $\% 201657156$.

${ }^{48}$ COSTA, A.C.; BORGES, M.S. op. cit., p. 77-89.

${ }^{49}$ TANAKA, Oswaldo Yoshimi. A judicialização da prescrição medicamentosa no SUS ou o desafio de garantir o direito constitucional de acesso à assistência farmacêutica. Revista de Direito Sanitário, v. 9, n. 1, p. 139143, mar./jun. 2008. https://doi.org/10.11606/issn.2316-9044.v9i1p139-143. Disponível em: http:// www.revistas.usp.br/rdisan/article/view/13109/14912.
} 
e, especialmente, a intensa luta social permitiram explicitar esses preceitos como norteadores da política sanitária do país. O que se identifica na presente pesquisa é a necessidade de se envidarem esforços para viabilizar a garantia desse direito em toda a sua amplitude.

Perante todo o discutido, oportuno registrar que o material analisado no presente estudo versa sobre casos situados, à época dos fatos, na esfera extrajudicial, cujas possibilidades de resolução perpassavam por diferentes instrumentos e estratégias de atuação ministeriais disponíveis: investigação, recomendação administrativa, termo de ajustamento de conduta (TAC), audiência pública, programas, entre outros. Somente uma parcela desse universo culminou com a propositura de uma ação civil pública, convertendo o feito à esfera forense. Não há informação sobre o quantitativo resultante nesse desfecho. Contudo, sabe-se que há, atualmente, tendência crescente da utilização de instrumentos extrajudiciais na resolução dos casos ${ }^{50,51}$.

\section{Considerações finais}

A pesquisa revelou o principal gargalo e fragilidade do SUS no Estado do Paraná a partir das denúncias ao Ministério Público estadual entre os anos de 2016 e 2017: o provimento de medicamentos. Outras debilidades também identificadas referem-se à gestão do sistema público de saúde, à insuficiência de leitos hospitalares, à má prática profissional e, em menor frequência, à saúde mental.

As hipóteses aventadas para explicar o motivo pelo qual os usuários do sistema público buscam guarida junto ao MP passam por fatores que incluem a afronta do poder público aos princípios constitucionais do SUS, a amplitude e a pretensão da política pública de assistência farmacêutica no país, a inabilidade dos gestores na resolução de conflitos e a inviabilidade de acesso a jurisconsulto particular.

Ante a constatação de que a problemática fundamental apontada pelo estudo está ligada ao fornecimento de fármacos e, por conseguinte, às limitações da universalidade e da integralidade do sistema sanitário público, foram debatidas as variantes imbricadas nesses preceitos: (i) a indefinição sobre os limites do dever do Estado no que se refere ao oferecimento integral de serviços e produtos; (ii) a extensão e a pretensão da política de assistência farmacêutica do SUS; (iii) os interesses conflitantes entre Estado e mercado, constituindo, por vezes, práticas deletérias; e (iv) os desafios regulatórios; entre outros. Para além de tal indicativo, o estudo sugere uma reflexão sobre o sistema de saúde do Estado do Paraná no tocante ao

\footnotetext{
${ }^{50}$ VIDAL, Denise da Silva. O Caduceu de Hermes: a atuação do Ministério Público Estadual junto aos Conselhos Municipais de Saúde no Estado do Rio de Janeiro. 2011. Dissertação (Mestrado em Ciências Jurídicas e Sociais) - Universidade Federal Fluminense, Niterói, 2011.

${ }^{51}$ MAPELLI JÚNIOR, Reynaldo. Ministério Público: atuação na área da saúde pública. In: SABELLA, Walter Paulo; DAL POZZO, Antônio Araldo Ferraz; BURLE FILHO, José Emmanuel (Coords.) Ministério Público: vinte e cinco anos no novo perfil constitucional. São Paulo: Malheiros, 2015. p. 547-584.
} 
reconhecimento de suas debilidades e à possibilidade de elaboração e deliberação próprios, de modo não só a desafogar o Judiciário e seus prepostos, como também a garantir a autonomia e a soberania do Executivo na resolução de conflitos.

Vale registrar que qualquer cidadão pode demandar questões em saúde, individuais ou coletivas, ao Ministério Público do Estado do Paraná. As solicitações podem ser efetivadas via contato telefônico, correspondência física, correio eletrônico e atendimento presencial, devendo ser direcionadas à Comarca do município de residência do demandante.

\section{Referências}

AITH, Fernando; BUJDOSO, Yasmin; NASCIMENTO, Paulo Roberto do; DALLARI, Sueli Gandolfi. Os princípios da universalidade e integralidade do SUS sob a perspectiva da política de doenças raras e da incorporação tecnológica. Revista de Direito Sanitário, v. 15, p. 10-39, jul. 2014. https://doi.org/10.11606/issn.2316-9044.v15i1p10-39. Disponível em: http://www. revistas.usp.br/rdisan/article/view/82804/85759.

ALMEIDA, Gregório Assagra. Direitos fundamentais e os principais fatores de legitimação social do Ministério Público no neoconstitucionalismo. In: ALMEIDA, Gregório Assagra; SOARES JÚNIOR, Jarbas (Coord.). Teoria Geral do Ministério Público. Belo Horizonte: Del Rey, 2013. (Coleção Ministério Público e Direitos Fundamentais).

ANDRADE, Mônica Viegas; NORONHA, K. V. M. S. Uma nota sobre o princípio da integralidade do SUS. In: BACHA, Edmar; SCHWARTZMAN, Simon (Orgs.). Brasil: a nova agenda social. Rio de Janeiro: LTC, 2010. v. 1, p. 94-103.

ASENSI, Felipe Dutra. Indo além da judicialização: o ministério público e a saúde no Brasil. Rio de Janeiro, RJ: Escola de Direito do Rio de Janeiro da Fundação Getúlio Vargas, Centro de Justiça e Sociedade, 2010. Disponível em http://bibliotecadigital.fgv.br/dspace/ handle/10438/10313.

ASSIS, Gilmar de. SUS para todos: breves reflexões jurídico-sociais: avanços e desafios. ALMEIDA, Gregório Assagra de; SOARES JÚNIOR, Jarbas; ASSIS, Gilmar de (Orgs.).

BARDIN, Laurence. Análise de conteúdo. São Paulo: Edições 70, LDA, 2011.

BRITO-SILVA, Keila; BEZERRA, Adriana Falangola Benjamin; TANAKA, Oswaldo Yoshimi. Direito à saúde e integralidade: uma discussão sobre os desafios e caminhos para sua efetivação. Interface (UNI/UNESP. Online), v. 16, n. 40, p. 249-260, 2012. http://dx.doi. org/10.1590/S1414-32832012005000014. Disponível em: http://www.scielo.br/pdf/icse/ v16n40/aop1812.pdf.

CANUT, L. Operacionalização do Sistema Único de Saúde e de sua assistência farmacêutica diante da judicialização: um estudo de caso no Município de São José/SC. Revista de Direito Sanitário, v. 18, n. 2, p. 62-91, 26 dez. 2017. https://doi.org/10.11606/issn.2316-9044. v18i2p62-91. Disponível em: https://www.revistas.usp.br/rdisan/article/view/142012. 
CARDOSO, Cosme Santana. Análise das demandas judiciais de medicamentos: uma abordagem da realidade atual do Distrito Federal. Revista Jurídica da Procuradoria-Geral do Distrito Federal, Distrito Federal, Brasília-DF, v. 39, n. 1, p. 11-30, 2014. Disponível em: http:// revista.pg.df.gov.br/index.php/RJPGDF/article/download/210/133.

CHIEFFI, Ana Luiza; BARATA, Rita Barradas. Ações judiciais: estratégia da indústria farmacêutica para introdução de novos medicamentos. Rev. Saúde Públ., São Paulo, v. 44, n. 3, p. 421-429, 2010. http://dx.doi.org/10.1590/S0034-89102010000300005. Disponível em: http://www.scielo.br/pdf/rsp/v44n3/05.pdf.

COSTA, A.C.; BORGES, M.S. A judicialização como acesso ao direito à saúde: considerações ao debate brasileiro. Sociedade em Debate, Pelotas, v. 16, n. 1, p. 77-89, 2012.

COSTA, Alexandre Bernardino et al. O Direito achado na rua: introdução crítica ao direito à saúde. Brasília-DF: CEAD/ UnB, Brasília, 2009.

DALLARI, Sueli Gandolfi. Aspectos particulares da chamada judicialização da saúde. Revista de Direito Sanitário, São Paulo, v. 14, n. 1, p. 77-81, jun. 2013. ISSN 2316-9044. https://doi. org/10.11606/issn.2316-9044.v14ilp77-81. Disponível em: http://www.revistas.usp.br/rdisan/ article/view/56624.

FIGUEIREDO, Tatiana Aragão. Análise dos medicamentos fornecidos por mandado judicial na Comarca do Rio de Janeiro: a aplicação de evidências científicas no processo de tomada de decisão. 2010. Dissertação (Mestrado em Saúde Pública). Escola Nacional de Saúde Pública Sérgio Arouca. Fundação Oswaldo Cruz, Rio de Janeiro, 2010.

FLICK, Uwe. Introdução à pesquisa qualitativa. Tradução Joice Elias Costa. 3. ed. Porto Alegre-RS: Editora Artmed, 2009.

FOCAULT, Michel. Microfísica do poder. 24. ed. São Paulo: Edição Graal, 2007.

GLOBEKNER, Osmir Antonio. A tutela individual e coletiva do direito à saúde pelo Ministério Público. Revista Jurídica da Escola Superior do Ministério Público de São Paulo, v. 8, p. 129-148, 2015. Disponível em: http://www.esmp.sp.gov.br/revista_esmp/index.php/RJESMPSP/article/ download/209/120.

GOMES, Valéria Bastos; PASSOS, Daniela Veloso Souza. A judicialização da saúde e as políticas públicas para fornecimento de medicamentos: uma análise a partir das decisões do TRF da 5 Região. Brazilian Journal of Empirical Legal Studies, Rio de Janeiro, v. 4, 2017. http://dx.doi.org/10.19092/reed.v4i3.168. Disponível em: https://www.researchgate. net/publication/321657139_A_JUDICIALIZACAO_DA_SAUDE_E_AS_POLITICAS_ PUBLICAS_PARA_FORNECIMENTO_DE_MEDICAMENTOS_UMA_ANALISE_A_ PARTIR_DAS_DECISOES_DO_TRF_DA_5_REGIAO.

LEITE, Silvana Nair; MAFRA, Ana Cristina Que direito? Trajetórias e percepções dos usuários no processo de acesso a medicamentos por mandados judiciais em Santa Catarina. Ciênc. saúde coletiva [online], v. 15, n. 1, p. 1665-1672, 2010. http://dx.doi.org/10.1590/S141381232010000700078. Disponível em: http://www.scielo.br/pdf/csc/v15s1/078.pdf. 
LOUREIRO, Maria Rita. As origens e consequências da judicialização da política. Resenha. Revista Brasileira Ciência Sociais, São Paulo, v. 29, n. 84, p. 189-199, fev. 2014. http://dx.doi. org/10.1590/S0102-69092014000100012. Disponível em: http://www.scielo.br/pdf/rbcsoc/ v29n84/11.pdf.

MACHADO Marina Amaral de Ávila et al. Judicialização do acesso a medicamentos no Estado de Minas Gerais, Brasil. Rev. saúde pública, v. 45, n. 3, p. 590-598, 2011. http://dx.doi. org/10.1590/S0034-89102011005000015. Disponível em: http://www.scielo.br/pdf/rsp/ v45n3/2403.pdf.

MACHADO, Marina Amaral de Ávila. Acesso a medicamentos via poder judiciário no Estado de Minas Gerais. 2010. Dissertação (Mestrado) - Faculdade de Farmácia, Universidade Federal de Minas Gerais, Belo Horizonte-MG, 2010.

MAGALHÃES, Iasmim Pacheco. Judicialização da saúde: critérios e limites para a atuação de magistrados em ações judiciais para aquisição de medicamentos pelo Estado. 2014. $65 \mathrm{f}$. Monografia (Bacharelado em Direito) - Universidade de Brasília, Brasília-DF, 2014. Disponível em: http://bdm.unb.br/bitstream/10483/10100/1/2014_IasmimPachecoMagalhaes.pdf.

MAGGIO, Marcelo P. A contribuição do Ministério Público à resolutiva implantação de políticas públicas de saúde: enfoque extrajudicial. Revista Jurídica do Ministério Público do Estado do Paraná, v. 1, p. 1-34, 2014. Disponível em: https://www. academia.edu/20947468/A_CONTRIBUI\%C3\%87\%C3\%83O_DO_MINIST\%C3\%89 RIO_P\%C3\%9ABLICO_\%C3\%80_RESOLUTIVA_IMPLANTA\%C3\%87\%C3\%83O_ DE_POL\%C3\%8DTICAS_P\%C3\%9ABLICAS_DE_SA\%C3\%9ADE_ENFOQUE_ EXTRAJUDICIAL_1.

MAGGIO, Marcelo P. Tutela da saúde pública: novas perspectivas e a construtiva atuação do Ministério Público. 2018. Tese (Doutorado em Serviços de Saúde Pública) - Faculdade de Saúde Pública, Universidade de São Paulo, São Paulo, 2018. Disponível em: http://www.teses. usp.br/teses/disponiveis/6/6135/tde-12042018-123251/.

MAPELLI JÚNIOR, Reynaldo. Ministério Público: atuação na área da saúde pública. In: SABELLA, Walter Paulo; DAL POZZO, Antônio Araldo Ferraz; BURLE FILHO, José Emmanuel (Coords.) Ministério Público: vinte e cinco anos no novo perfil constitucional. São Paulo: Malheiros, 2015. p. 547-584.

MELLO, Alexandra Ferro de et al. Uma abordagem econômica de processos judiciais de medicamentos impetrados contra um município do sul do Brasil. Jornal Brasileiro de Economia da Saúde, v. 8, n. 1, p. 39-46, 2016. http://dx.doi.org/ 10.21115/JBES.v8.n1.p39-46. Disponível em: http://files.bvs.br/upload/S/2175-2095/2016/v8n1/a5479.pdf.

NAKAMURA, Fernanda de Castro. A judicialização da saúde e a atuação do Ministério Público no sistema de fornecimento gratuito de medicamentos na região de Ribeirão Preto. 2017. Dissertação (Mestrado em Planejamento a Análises de Políticas Públicas) - Universidade Estadual Paulista Júlio de Mesquita Filho, Franca, 2017. 
NORONHA, José Carvalho de; LIMA, Luciana Dias de; MACHADO, Cristiani Vieira. O Sistema Único de Saúde - SUS. In: GIOVANELLA, Ligia et al (Orgs.). Políticas e sistemas de saúde no Brasil. Rio de Janeiro: Ed. FIOCRUZ, 2012. p. 365-393.

OLIVEIRA, Denize Cristina de Análise de conteúdo temático-categorial: uma proposta de sistematização. Revista Enfermagem UERJ, Rio de Janeiro, v. 16, p. 569-76, 2008. Disponível em: http://www.facenf.uerj.br/v16n4/v16n4a19.pdf.

OLIVEIRA, Maria dos Remédios Mendes et al. Judicialização da saúde: para onde caminham as produções científicas? Saúde debate [online], Rio de Janeiro, v. 39, n. 105, p. 525-535, 2015. http://dx.doi.org/10.1590/0103-110420151050002019. Disponível em: http://www.scielo.br/ pdf/sdeb/v39n105/0103-1104-sdeb-39-105-00525.pdf.

PAIM, Jairnilson Silva. Uma análise sobre o processo da Reforma Sanitária brasileira. Saúde em Debate, Rio de Janeiro, v. 33, n. 81, p. 27-37, jan./abr. 2009. Disponível em: https://repositorio. ufba.br/ri/bitstream/ri/5978/1/Paim\%20JS\%202009.\%20Artigo2.pdf.

PAULA, Cléria Maria Lobo; BITTAR, Cléria Maria Lobo. Judicialização da saúde e seus reflexos na gestão do sistema único de saúde (SUS). Revista de Direitos Humanos e Efetividade, v. 3, p. 19-41, 2017. Disponível em: https://www.researchgate.net/publication/322100237_ Judicializacao_da_saude_e_seus_reflexos_na_gestao_do_Sistema_Unico_de_Saude_SUS.

PEPE, V. L. E. et al. A judicialização da saúde e os novos desafios da gestão da assistência farmacêutica. Ciência e Saúde Coletiva, (Impresso), v. 15, n. 5, p. 2405-2414, 2010.

RELATÓRIOS de recomendação da Conitec. CONITEC. Comissão Nacional de Incorporação de Tecnologias no Sistema Único de Saúde, 29 jul. 2014. Disponível em: http://conitec.gov. br/relatorios-de-recomendacao-da-conitec.

REZENDE, Conceição Aparecida Pereira; PEIXOTO, Maria Passos Barcala. Metodologia para análises funcionais da gestão de sistemas e redes de serviços de saúde no Brasil. 1. ed. Brasília-DF: Organização Pan-Americana da Saúde (OPAS), 2003. v. 1.

RODRIGUES, João Gaspar. A nova dinâmica resolutiva do Ministério Público. Jus Navegandi, ago.2014. Disponível em: https://jus.com.br/artigos/30584/a-nova-dinamica-resolutiva-doministerio-publico. Acesso em: 08 mar. 2018.

ROTUNNO, Angela Salton. Judicialização e juridicização da saúde no Ministério Público. In: ASENSI, Felipe Dutra; PINHEIRO, Roseni (Orgs.). Direito sanitário. Rio de Janeiro: Elsevier, 2012. p. 517-531.

SANTOS, Lenir. Judicialização da saúde e a incompreensão do SUS. In: SANTOS, Lenir; TERRAZAS, Fernanda (Orgs.) Judicialização da saúde no Brasil. 1. ed. Campinas: Saberes Ed., 2014.

SARLET, Ingo Wolgang; SAAVEDRA, Giovani Agostini. Judicialização, reserva do possível e compliance na área da saúde. Revista de Direitos e Garantias Fundamentais, Vitória, v. 18, n. 1, p. 257-282, jan./abr. 2017. 
SMITH, Richard. Medical journals are an extension of the marketing arm of pharmaceutical companies. PLoSMed, v. 2, n. 5, p. 138, 2015. https://doi.org/10.1371/journal.pmed.0020138. Disponível em: https://journals.plos.org/plosmedicine/article/file?id=10.1371/journal. pmed.0020138\&type $=$ printable.

SOUZA NETO, Claudio Pereira de; SARMENTO, Daniel (Coords.). Direitos sociais: fundamentos, judicialização e direitos sociais em espécie. Rio de Janeiro: Lumen Juris, 2008. p. 313-339.

TANAKA, Oswaldo Yoshimi. A judicialização da prescrição medicamentosa no SUS ou o desafio de garantir o direito constitucional de acesso à assistência farmacêutica. Revista de Direito Sanitário, v. 9, n. 1, p. 139-143, mar./jun. 2008. https://doi.org/10.11606/issn.2316-9044. v9i1p139-143. Disponível em: http://www.revistas.usp.br/rdisan/article/view/13109/14912.

VIDAL, Denise da Silva. O Caduceu de Hermes: a atuação do Ministério Público Estadual junto aos Conselhos Municipais de Saúde no Estado do Rio de Janeiro. 2011. Dissertação (Mestrado em Ciências Jurídicas e Sociais) - Universidade Federal Fluminense, Niterói, 2011.

Suéllyn Mattos de Aragão - Mestrado em Saúde Coletiva pela Universidade Federal do Paraná (UFPR). Médica servidora da UFPR e do Ministério Público do Estado do Paraná. Curitiba/PR, Brasil.E-mail: smdaragao@mppr.mp.br

André Luis Cândido da Silva - Mestrado em Saúde Coletiva pela Universidade Federal do Paraná (UFPR). Farmacêutico servidor da Secretaria de Saúde do Estado do Paraná. Curitiba/PR, Brasil. E-mail: silvacandre@gmail.com

Rafael Gomes Ditterich - Doutorado em Saúde Coletiva pela Pontifícia Universidade Católica do Paraná (PUC-PR). Professor adjunto da Universidade Federal do Paraná (UFPR). Curitiba/PR, Brasil. E-mail: prof.rafaelgd@gmail.com

Solena Ziemer Kusma - Doutorado em Saúde Coletiva pela Pontifícia Universidade Católica do Paraná (PUC-PR); mestrado em Saúde Coletiva pela University College London (Inglaterra). Professora adjunta da Universidade Federal do Paraná (UFPR). Curitiba/PR, Brasil.E-mail: solenakusma@gmail.com 